\title{
La vidéocapsule digestive cinq ans après la commission technique à la HAS et trois ans après sa prise en charge : doit-on rediscuter la prise en charge de nouvelles indications?
}

\author{
Video capsule endoscopy five years on from the establishment of the technical commission \\ of the HAS (French National Authority for Health) and three years after obtaining cover \\ for the technique under the national health insurance system: should we be considering such \\ cover for further indications?
}

\author{
D. Heresbach \\ (C) Springer-Verlag France 2010
}

Point n'est besoin de refaire l'historique de la vidéocapsule digestive (VD), initialement de la vidéocapsule du grêle (VCG), puis de la vidéocapsule œsophagienne (VCO), et actuellement en cours d'évaluation au niveau colique (VCC). Cet exemple a démontré à la communauté gastroentérologique le long parcours scientifique, médical, administratif et économique de la mise à disposition d'une procédure destinée à l'exploration d'un segment digestif qui, il n'y a pas si longtemps encore, demeurait une zone quasi aveugle.

Le hasard veut que la commission capsule de la SFED depuis un an reprenne les données, publiées en particulier sur les indications qui n'avaient pas été retenues initialement en 2006, afin de nous faire bénéficier d'une mise au point synthétique dans ce numéro, et que, de façon concomitante et indépendante, l'European Society of Gastrointestinal Endoscopy (ESGE) publie dans le numéro de mars 2010 d'Endoscopy [1] ses recommandations pour l'exploration par VCG, VCO et VCC.

Il est donc possible de vérifier si en France nous sommes « aux normes européennes » et dans quel domaine il demeure des écarts d'ordre scientifique ou économique. Cet exercice est d'autant plus pertinent que les 27 et 28 août 2010 se tiendra, à Paris, la première rencontre ICCD regroupant l'ensemble des experts mondiaux de la VCD et de l'endoscopie double ballon (EDB).

Les recommandations de L'ESGE, élaborées selon la méthode d'Oxford pour l'Evidence-Based Medicine, com-

\section{Heresbach $(\square)$}

Rédacteur en chef d'Acta Endoscopica

Service des maladies de l'appareil digestif, CHU Pontchaillou,

2, rue Henri-Le-Guilloux, F-35033 Rennes cedex 09, France

e-mail : denis.heresbach@chu-rennes.fr portent quatre grades de recommandation (A à D) couplés à huit catégories de niveau de preuve $(1 \mathrm{a}, 1 \mathrm{~b}, 2 \mathrm{a}, 2 \mathrm{~b}, 3 \mathrm{a}, 3 \mathrm{~b}$, 4 et 5) ou d'évidence (E). Elles abordent initialement la question de la préparation à l'examen par VCG en recommandant une préparation purgative qui augmente le rendement diagnostique sans améliorer le caractère complet de l'exploration du grêle (E2a, gB). Pour les saignements digestifs inexpliqués, la VCG est l'examen de première ligne après une exploration par fibroscopie haute et coloscopie négative $(\mathrm{E} 2 \mathrm{~b}, \mathrm{gB})$ ainsi que pour les anémies ferriprives inexpliquées (E2b, gB). Au cours de la maladie de Crohn (MC), la VCG est

le meilleur examen pour apprécier les lésions muqueuses de la $\mathrm{MC}(\mathrm{E} 3 \mathrm{a}, \mathrm{gB})$; mais en cas de MC avérée et connue, le risque de blocage nécessite un autre moyen d'imagerie du grêle préalable ou la réalisation d'une capsule patency (E2b, gB). En cas de prise d'anti-inflammatoires, l'exploration par VCG doit être réalisée deux mois après leur arrêt (E2a, gB). Au cours de la maladie cœliaque, la VCG possède un fort rendement diagnostique et doit être réalisée en cas de biopsies négatives $(\mathrm{E} 2 \mathrm{~b}, \mathrm{gB})$ ou de forme réfractaire ou compliquée (jéjuno-iléite diffuse, lymphome du grêle). Lors des polyposes atteignant le grêle, la VCG doit être réalisée en première ligne pour la surveillance des patients atteints du syndrome de Peutz-Jeghers (E2b, gB) et est souhaitable au cours des polyposes adénomateuses familiales avec adénomes duodénaux (E2a, gB). L'exploration par VCG dans la stratégie de prise en charge des tumeurs du grêle peut être indiquée $(\mathrm{E} 3 \mathrm{~b}, \mathrm{gB})$. Enfin, la VCO présente une bonne concordance avec la fibroscopie œsogastrique pour le diagnostic d'œsophage de Barrett et de varices œsophagiennes (E2a, gB). Le résumé des possibilités de la VCC est rappelé mais ne fait pas l'objet de recommandation, en 2010. 
Les avis d'experts formulés par la commission vidéocapsule de la SFED relèvent d'une méthode moins coercitive se rapprochant de la méthode de Delphes, bien qu'un avis final collégial n'ait pas été rendu. Ils concernaient les thèmes suivants : saignement digestif inexpliqué (Dr A. De Leusse), la maladie de Rendu-Osler (Pr G. Gay), la MC (Drs A. Attar, V. Maunoury), la maladie cœliaque ( $\operatorname{Pr}$ C. Cellier), les polyposes du grêle (Pr J.-C. Saurin), les douleurs abdominales (Dr M. Delvaux) et la VCC (Pr B. Filoche). Il est utile, à ce stade, de préciser que les indications reconnues par la HAS donnant droit à remboursement sont les saignements digestifs inexpliqués après endoscopie haute et iléocoloscopie négatives et la suspicion de $\mathrm{MC}$ après explorations traditionnelles négatives. Les éléments nouveaux par rapport aux indications retenues par la HAS apportés par les experts de la SFED sont :

- pas d'indication au cours de la maladie de Rendu-Osler en dehors d'anémies inexpliquées — en particulier inexpliquées par des épistaxis. La surveillance après traitement endoscopique de lésions digestives n'a pas fait l'objet de publications permettant de juger de sa pertinence ;

- indications au cours de la maladie cœliaque en cas de biopsies impossibles par troubles persistants de la coagulation ou de négativité des biopsies duodénales en présence d'anticorps antitransglutaminase ou antiendomysium (et non antigliadine) ;
- indication recommandée de VCG au cours de la surveillance des polyposes de Peutz-Jeghers sans pouvoir déterminer avec certitude le rythme (tous les deux ans ?), mais pas de recommandations au cours de la polypose adénomateuse familiale avérée en dehors des anémies inexpliquées, et recommandation souhaitable d'un dépistage des carcinomes et/ou des adénomes du grêle au cours du syndrome de Lynch sans pouvoir préciser l'âge et le rythme de sa réalisation ;

- indication possible au cours de douleurs abdominales chroniques (délai ?), plus marquée en cas de diarrhée ou de signes d'alarme associés avec un niveau de preuve faible;

- pas d'indication recommandée pour la VCC.

Parions que l'ICCD de Paris 2010 permettra de donner un éclairage sur ces questions sans réponses et de préciser la place respective du couple VCG-EDB avant d'entamer le marathon de la procédure, PHRC-STIC-HAS-CNAM(ARS?)!

Rendez-vous dans dix ans?

\section{Référence}

1. Ladas SD, Triantafyllou K, Spada C, et al. European Society of Gastrointestinal Endoscopy (ESGE): recommendations (2009) on clinical use of video capsule endoscopy to investigate smallbowel, esophageal and colonic diseases. Endoscopy 2010;42:220-7. 